\title{
Arylthio-substituted coronenes as tailored building blocks for molecular electronics $\dagger$
}

\author{
Peter Kowalzik, ${ }^{a}$ Nicolae Atodiresei, $\ddagger^{b}$ Marc Gingras,,$\S^{c}$ Vasile Caciuc, ${ }^{b}$ \\ Nicolas Schnaebele, ${ }^{d}$ Jean-Manuel Raimundo, ${ }^{c}$ Stefan Blügel, ${ }^{b}$ Rainer Waser ${ }^{a}$ and \\ Silvia Karthäuser $\uparrow * a$
}

Received 14th October 2011, Accepted 28th November 2011

DOI: $10.1039 / \mathrm{c} 2 \mathrm{cp23241c}$

The electron transport through molecules in molecular devices is typically influenced by the nature of the interfaces with the contacting electrodes and by the interactions between neighbouring molecules. It is a major goal of molecular electronics to adjust the electronic function of a molecular device by tailoring the intrinsic molecular properties and the interfacial and intermolecular interactions. Here, we report on the tunability of the electronic properties of coronene derivatives, namely dodecakis(arylthio)coronenes (DATCs), which are found to exhibit a three-dimensional aromatic system. Scanning tunnelling microscopy (STM), spectroscopy (STS) and simulations based on the density functional theory (DFT) are employed to characterize the structural and electronic properties of these molecules deposited on $\mathrm{Au}(111)$ surfaces. It is shown that modifications of the peripheral aryl-groups allow us to specifically affect the self-assembly and the charge transport characteristics of the molecules. Molecular assemblies like supramolecular wires with highly delocalized orbitals and single molecules with molecular "quantum dot" characteristics are obtained in this way.

\section{Introduction}

The development of future nanoelectronic devices based on single molecules or well-defined molecular assemblies is driven by the vision that a great variety of functionalities may be implemented by using the appropriate chemical design. ${ }^{1,2}$ In the case of single molecule junctions, the charge injection and transport is naturally affected by the chemical structure of the molecule, but additionally the property of the electrodemolecule interface plays a significant or even dominant role. ${ }^{3}$ The performance of organic electronic devices based on thin films or supramolecular architectures, on the other hand, crucially depends on the efficiency of the charge transport through the organic layer(s). ${ }^{4,5}$ In this context, the existence of

${ }^{a}$ Peter Grünberg Institut (PGI-7) and JARA-FIT,

Forschungszentrum Jülich GmbH, 52425 Jülich, Germany.

E-mail: s.karthaeuser@fz-juelich.de

${ }^{b}$ Peter Grünberg Institut (PGI-1) and Institute for Advanced

Simulation, Forschungszentrum Jülich GmbH, 52425 Jülich,

Germany

${ }^{c}$ CNRS, Aix-Marseille University, UPR 3118 CINAM,

13288 Marseille Cedex 09, France

${ }^{d}$ University of Nice-Sophia Antipolis, 06108 Nice Cedex 2, France

$\dagger$ Electronic supplementary information (ESI) available: Analytical data of the DATC derivatives. See DOI: 10.1039/c2cp23241c

$\ddagger$ Corresponding author for $A b$ initio calculations.

$\S$ Corresponding author for organic synthesis and supramolecular chemistry.

- Corresponding author for STM and electron transport studies. delocalized molecular orbitals throughout the whole molecule and sufficiently strong intermolecular interactions are the basis of conduction in organic semiconductors.

In this study, we report on a molecular system based on a polycyclic aromatic hydrocarbon (PAH) core, more specifically coronene. A thorough phenylthio-substitution of coronene leads to dodecakis(phenylthio)coronene (DPTC), which is known to exhibit several unique (opto)electronic properties. ${ }^{6}$ For instance, earlier spectroelectrochemical studies revealed a greater stabilization of the charged DPTC species, pointing to a larger delocalization of molecular orbitals, when compared to the non-substituted coronene. ${ }^{7,8}$ These findings in combination with the non-planar molecular structure suggest that such molecules can be seen as nanosized systems with a three-dimensional delocalized electronic network.

Here, we focus on the self-assembly behaviour and the electronic properties of systematically varied dodecakis(arylthio)coronenes (DATCs) derivatives, studied by STM/ STS measurements and theoretically investigated by firstprinciples calculations. It will be shown how the self-assembly of DATCs on $\mathrm{Au}(111)$ can be specifically directed into different regimes by using appropriate substituted arylthio moieties. A broad range of different electronic functionalities can be engineered along with the adsorption behaviour on the surface and the modification of the supramolecular structure. 


\section{Methods and materials}

\subsection{Synthesis}

The synthesis of dodecakis[p-(trifluoromethyl)phenylthio]coronene (DFPTC), dodecakis(phenylthio)coronene (DPTC), and dodecakis( $p$-methoxyphenylthio)coronene (DMPTC), respectively, was based on the sulfuration of perchlorocoronene as a precursor. The synthetic preparation of perchlorocoronene was previously described from the chlorination of coronene in a quantitative yield. ${ }^{9}$ DFPTC, DPTC, and DMPTC were prepared similarly to a previously published procedure for dodecakis( $p$-methylphenylthio)coronene. ${ }^{7}$ As a typical procedure for all coronene derivatives, the synthesis of DFPTC is described. Analytical data of the DATC derivatives under investigation will be reported in the ESI. $\dagger$

In an oven-dried two-neck round bottom flask was weighed dodecachlorocoronene (50 mg, $0.07 \mathrm{mmol}, 1.0$ eq.) and 4-trifluoromethylbenzenethiol (165 mg; $0.92 \mathrm{mmol}, 13.2 \mathrm{eq}$.) or corresponding amounts of thiophenol or 4-methoxybenzenethiol, respectively, under a nitrogen atmosphere. 1,3-Dimethyl2-imidazolidinone dried over activated $3 \AA$ sieves $(1.5 \mathrm{~mL})$ was injected via a syringe and the flask was cooled in an ice-bath at $3{ }^{\circ} \mathrm{C}$. Powdered sodium hydride (40 mg, $1.68 \mathrm{mmol}, 24.0$ eq.) was slowly added in small portions via a lateral Gooch tube connected to the main flask while vigorously stirring the mixture. The ice-bath was removed after completing the addition of $\mathrm{NaH}$ and stirring was continued for 2 hours at $20{ }^{\circ} \mathrm{C}$ (4 hours for DMPTC). While stirring, a $\mathrm{NaOH}$ solution ( $1 \mathrm{M}, 20 \mathrm{~mL}$ ) was slowly added. The aqueous phase was then extracted with toluene and the combined organic layers were kept. The organic phase was then washed with water and dried over anhydrous $\mathrm{Na}_{2} \mathrm{SO}_{4}$. Filtration, evaporation of the solvent and careful drying under high vacuum afforded: DFPTC (orange amorphous powder, 58\% yield); DPTC (deep red powder, 69\% yield); and DMPTC (deep red amorphous powder, 95\% yield). The corresponding analytical data for DFPTC, DPTC and DMPTC, respectively, are given in the ESI. $\dagger$

\subsection{Experimental techniques}

The scanning tunneling microscopy (STM) and spectroscopy (STS) experiments were carried out with a commercial JEOL $4500 \mathrm{~S}$ STM head operated in ultra-high vacuum at room temperature (RT). Probe tips were electrochemically etched from tungsten wires. The (111)-oriented gold thin films were fabricated by electron beam evaporation of gold on mica in a two step deposition process. ${ }^{10}$ A drop of a diluted solution (concentration $c \approx 10^{-6} \mathrm{~mol} \mathrm{~L}^{-1}$ ) of DATCs in toluene was deposited onto the freshly prepared substrates, resulting in a nearly one monolayer coverage. After complete solvent evaporation, the samples were immediately transferred into the UHV-STM measurement chamber. STM images were obtained in constant current mode. The sample voltage and the tunneling current used during image acquisition are denoted as $V_{\mathrm{s}}$ and $I_{\mathrm{t}}$, respectively. Positive voltages mean that electrons tunnel from the tip into unoccupied states of the sample. Tunneling current versus voltage curves, $I(V)$, were recorded with 128 equidistant bias voltage steps after switching off the feedback loop. The tip-sample distance was adjusted according to the established current set point $I_{\mathrm{t}}$ at a given voltage set point $V_{\mathrm{s}} \cdot \mathrm{d} I / \mathrm{d} V$ spectra were obtained from differentiation of an average of 20 single $I(V)$ traces.

\subsection{Computational methods}

By means of $a b$ initio calculations we investigated the geometry and electronic structure of several conformers of the isolated DATC molecules. In our study, the first-principles totalenergy calculations have been performed in the framework of the density-functional theory (DFT) as implemented in the pseudopotential plane wave VASP code. ${ }^{11,12}$ The electron-ion interactions are described by pseudopotentials obtained with the projector-augmented wave (PAW) method. ${ }^{13,14}$ As DFT exchange-correlation energy functional we used the generalized gradient approximation (GGA) as parameterized by Perdew, Burke and Ernzerhof (PBE). ${ }^{15}$ To account for the effect of the long-range van der Waals interactions on the geometry and total energy of the DATC conformers we employed the semiempirical approach proposed by Grimme. ${ }^{16}$ In this method the semi-empirical energy and force corrections derived from a damped atom-pairwise potential $V(R)=C_{6} R^{-6}\left(C_{6}\right.$ represents the dispersion coefficient for a given atom pair and $R$ is the distance between the atoms) are added during the self-consistent cycle of the DFT-GGA calculations. Previously this semiempirical method was successfully used to describe the flat adsorption of molecules like benzene, pyridine and pyrazine on a $\mathrm{Cu}(110)$ surface. $^{17}$

\section{Results and discussion}

\subsection{Structural characteristics of DATCs on Au(111)}

We investigated dodecakis(arylthio)coronenes (DATCs) with three different functionalities, which are shown in Fig. 1. The chemical structure of these compounds is systematically modified by use of arylthio-moieties with different substituents in the para-position, i.e. the electron-withdrawing $p$-(trifluoromethyl) phenylthio-moiety, the phenylthio-moiety, and the electron-donating $p$-methoxyphenylthio-moiety leading to the compounds DFPTC, DPTC, and DMPTC, respectively.

The surface morphology obtained after depositing DFPTC on $\mathrm{Au}(111)$ as a monolayer is shown in the STM image of Fig. 2(a). The molecules form ordered domains consisting of parallel chains. These self-assembled columnar domains are surrounded by a disordered layer. Fig. 2(c) displays a high

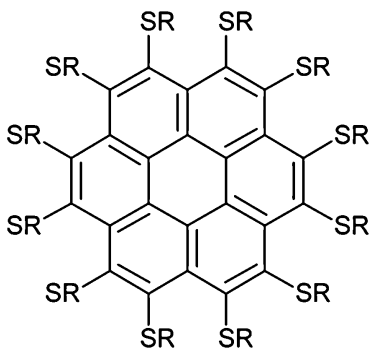<smiles>[R]=[PH2]c1ccc(OC)cc1</smiles>

Fig. 1 Chemical structure of the investigated DATC molecules: dodecakis[ $p$-(trifluoromethyl)phenylthio]coronene (DFPTC), dodecakis(phenylthio)coronene (DPTC), and dodecakis( $p$-methoxyphenylthio)coronene (DMPTC). 
(a)

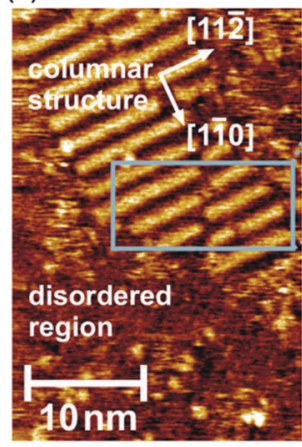

(b)

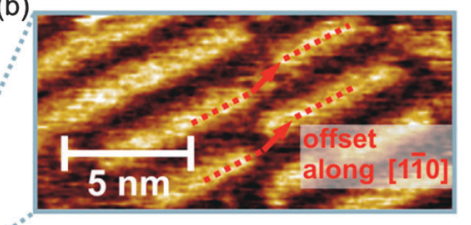

(c)

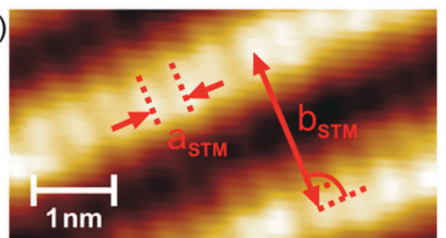

Fig. 2 (a) STM image $\left(V_{\mathrm{s}}=1.0 \mathrm{~V}, I_{\mathrm{t}}=0.12 \mathrm{nA}\right)$ of a DFPTC monolayer on $\mathrm{Au}(111)$. The molecules are observed to form supramolecular columns along the [112] direction of the underlying gold surface. (b) Zoom-in showing columns which are offset vertical to the growth direction by an integer multiple of the $\mathrm{Au}(111)$ lattice constant. (c) High resolution image of the structure. The distance between bright intracolumnar features has the average value $a_{\mathrm{STM}}=(0.47 \pm 0.05) \mathrm{nm}$. The intercolumnar spacing is $b_{\mathrm{STM}}=(2.24 \pm 0.20) \mathrm{nm}$.

resolution image of the chain structure with protruding features at distances of $a_{\mathrm{STM}}=(0.47 \pm 0.05) \mathrm{nm}$. This points to a growth of the chains along the [112] direction of the underlying $\mathrm{Au}(111)$ surface having a next-nearest-neighbour distance of $\sqrt{ } 3 a_{\mathrm{Au}(111)}=0.499 \mathrm{~nm}$, where $a_{\mathrm{Au}(111)}=0.288 \mathrm{~nm}$ is the lattice constant of the gold surface. The separation between adjacent chains amounts to $b_{\mathrm{STM}}=(2.24 \pm 0.20) \mathrm{nm}$. In Fig. 2(b) a zoom-in into the STM image of Fig. 2(a) is depicted. Some columns are interrupted and continue to grow in the same direction with a small shift in the [11̄0] direction. The displacement is equal to or an integer multiple of the $\mathrm{Au}(111)$ lattice constant and thus also points to a growth of the DFPTC columns commensurable to the substrate surface. Furthermore, this is supported by the observation that the columns in neighbouring domains are aligned parallel to each other or enclose an angle of $120^{\circ}$, reflecting the hexagonal symmetry of the $\mathrm{Au}(111)$ surface.

Moreover, the number of ordered domains and the domain sizes are observed to increase during storage in the UHV chamber at RT. This is exemplified in Fig. 3, which shows two consecutively recorded STM images. Molecules in the disordered regions around the columnar structure are observed to diffuse at RT and aggregate at the ends of the columns. It is seen that a new column begins to grow at the upper end of the domain and the column at the bottom of the image grows further.

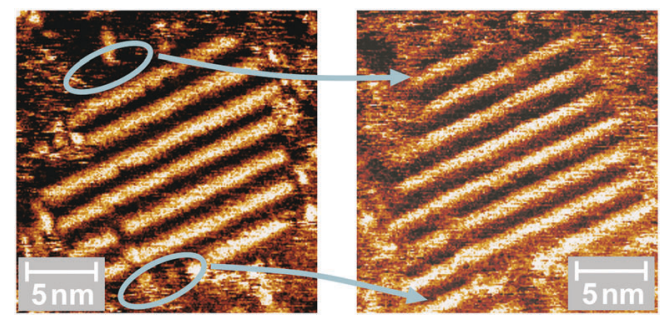

Fig. 3 Consecutively recorded STM images (3 min time difference between scan start) showing the proceeding growth of the molecules at room temperature. The ovals indicate regions in which columns are developing.

(a)

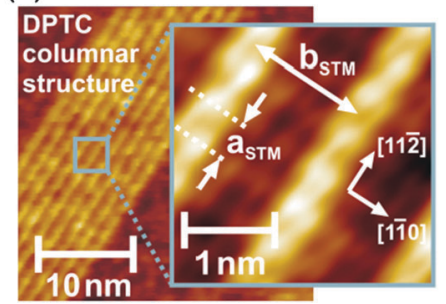

(b)

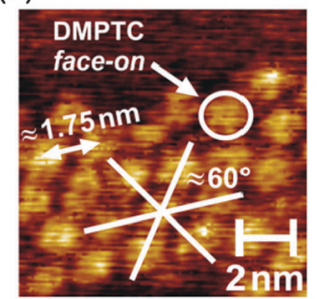

Fig. 4 (a) DPTC molecules assemble into a columnar structure similar to that of DFPTC. However, the intercolumnar separation is considerably smaller $\left(b_{\mathrm{STM}}=(1.44 \pm 0.20 \mathrm{~nm})\right)$. Furthermore, a greatly reduced tendency to form ordered domains is observed. (b) The self-assembly behaviour is entirely changed for DMPTC, which exhibits an arrangement of molecules in a face-on configuration (coronene core oriented parallel to the surface plane).

The columnar arrangement of DFPTC, a coronene derivative with twelve electron-withdrawing substituents, is in line with findings made in the framework of the related field of discotic liquid crystals. ${ }^{18,19}$ Molecules with strongly electronwithdrawing groups form stable columnar mesophases over a broad temperature range, while their analogues with electrondonating substituents directly melt from the crystalline state to the isotropic liquid. These observations are explained by the stabilization of a $\pi-\pi$-stacking between discotic aromatic molecules due to the fact that electron-withdrawing groups help to minimize the repulsive interactions between adjacent aromatic $\pi$-systems. ${ }^{20}$

A comparable structural arrangement is observed in the case of the molecular building block DPTC. As shown in Fig. 4(a), DPTC molecules also form domains with an ordered columnar structure. ${ }^{21}$ From higher resolution images the distance between protruding intracolumnar features is determined to be $a_{\text {STM }}=$ $(0.49 \pm 0.05) \mathrm{nm}$. Within the error margins this is equivalent to the distance of protrusions observed in the DFPTC columns. On the other hand, the intercolumnar separation $b_{\mathrm{STM}}=$ $(1.44 \pm 0.20) \mathrm{nm}$ is considerably smaller for DPTC than in the DFPTC structure. At first glance, the larger separation of the DFPTC columns is most probably due to steric effects caused by the additional space requirement of the $\mathrm{CF}_{3}$ groups.

Another striking difference comparing the self-assembly behaviour is the greatly enhanced tendency of DFPTC to form a well-ordered columnar structure in contrast to DPTC. The fraction of the surface covered with ordered columnar domains is $\sim 50 \%$ in the case of DFPTC, and $\sim 10 \%$ for DPTC. The columnar structures of both, DFPTC and DPTC, can be described by a stacked arrangement of molecules with an edge-on orientation.

A completely different self-assembly behaviour is observed for the coronene derivative with $p$-methoxyphenylthiomoieties, i.e. DMPTC. Here, no columnar phase could be observed for monolayers deposited on $\mathrm{Au}(111)$. The STM images in Fig. 4(b) show that the molecules seem to lie on the surface in a face-on configuration with the coronene core oriented parallel to the surface. The magnified STM image of Fig. 4(b) reveals that DMPTCs form a monolayer structure with hexagonal ordering on a short range and an intermolecular distance of $(1.75 \pm 0.20) \mathrm{nm} .{ }^{22}$ 
The STM topographical findings show that the self-assembly of the DATC system is significantly influenced by using different aryl-substituents. While the $\mathrm{CF}_{3}$ groups strongly enhance the tendency to form a chain structure, the $\mathrm{OCH}_{3}$ groups seem to anticipate the stacking into such a columnar phase. This behaviour is clearly correlated to the tendency of discotic molecules to form stable columnar mesophases if electron-withdrawing substituents are present. ${ }^{18-20}$

However, to find the same behaviour in the case of DATC molecules is rather surprising since these molecules are expected to form three dimensional conformers, in contrast to merely planar PAHs. The peripheral aryl groups of DATCs are forced to adopt a position above or below the plane of the coronene core due to the tetrahedral angle of the $\mathrm{sp}^{3}$ hybridized sulfur atoms and the space requirements of the aryl rings themselves, resulting in an overall non-planar geometrical structure. Consequently the coronene core will be geometrically shielded effectively by the arylthio-moieties, so that intermolecular coronene-coronene interactions are not possible.

To gain insight into the detailed molecular structure and possible intermolecular interactions, respectively, we performed DFT calculations including long range van der Waals (vdW) interactions. In the first instance, we investigated the geometrical structure and the stability of several conformers of the isolated single molecules. We have considered three different conformations: (i) in the up-down conformer adjacent aryl groups alternate between the position above and below the plane of the coronene unit, (ii) in the 2up-2down conformer alternately two adjacent aryl groups are located above and below the plane, and (iii) in the $u p-u p$ conformer all 12 aryl groups are located above the coronene plane. We optimized the geometry of each conformer in the gas phase by relaxing all atoms using an orthorhombic supercell with dimensions of $2.7 \times 2.7 \times 2.7 \mathrm{~nm}^{3}$. The relaxed molecular geometries were achieved when the sum of the calculated Hellmann-Feynman and $\mathrm{vdW}$ forces was smaller than a threshold of $0.01 \mathrm{eV} \mathrm{nm}^{-1}$. To evaluate such accurate forces, the plane wave basis set consists of all plane waves up to a cutoff energy $E_{\text {cut }}$ of $500.0 \mathrm{eV}$. Due to the large size of the supercells used in our $a b$ initio simulations, the Brillouin zone was sampled at the $\Gamma$ point only. In Fig. 5 the optimized geometries of stable conformers, i.e. conformers with a total energy corresponding to a local minimum, are plotted as top and side views for the case of the DFPTC molecule. Comparable geometries for the

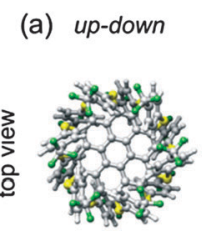

(b) 2up-2down

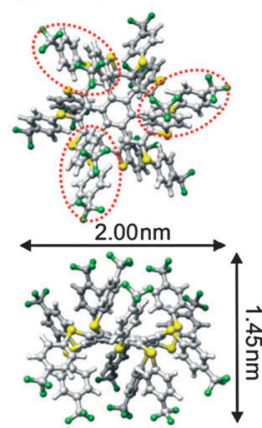

(c) up-up

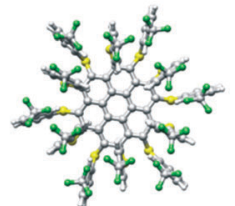

$1.98 \mathrm{~nm}$

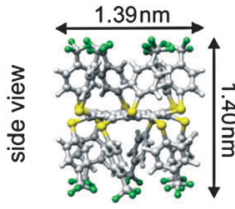

त क

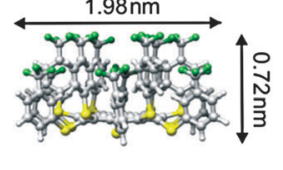

Fig. 5 Top and side views of the optimized geometries of the DFPTC conformers: (a) up-down, (b) $2 u p-2 d o w n$, and (c) up-up conformer.
Table 1 Total energies in eV of the different DATC conformers ${ }^{a}$

\begin{tabular}{lccc}
\hline & Up-down & 2up-2down & $U p-u p$ \\
\hline DFPTC & +0.16 & 0 & +0.77 \\
DPTC & 0 & +0.19 & +0.68 \\
DMPTC & 0 & +1.15 & +1.18
\end{tabular}

${ }^{a}$ The total energy of the most stable conformer is set to the reference value $0 \mathrm{eV}$ for all three DATC molecules.

up-down, 2up-2down, and up-up conformer are obtained for DPTC and DMPTC, respectively.

The $a b$ initio calculations reveal that the $u p-u p$ conformer of all investigated DATCs is significantly less stable than the other two conformers (Table 1). This suggests that the columnar structures should consist of molecules having a conformation with an alternating pattern of the substituents above and below the coronene core, that is a conformation close to up-down or 2up-2down. During the aggregation into the columnar structure, a geometrical reorganization of the peripheral groups is expected to occur and enable a commensurable growth on the $\mathrm{Au}(111)$ surface. As illustrated in Fig. 6, the edge-on molecules possess protruding aryl groups on each side of the coronene core, which therefore can be ascribed to the bright STM features along the columns. The intracolumnar distance between neighbouring molecules amounts to $a=$ $2 a_{\text {STM }}$. Accordingly, the STM measurements suggest a molecular overlayer structure having a $(8 \times 2 \sqrt{ } 3)$ and a $(5 \times 2 \sqrt{ } 3)$ rectangular unit cell in the case of DFPTC and DPTC, (a)

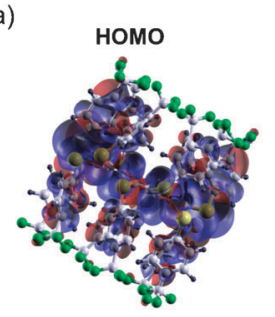

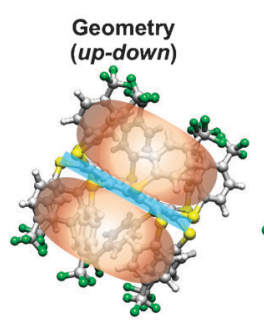

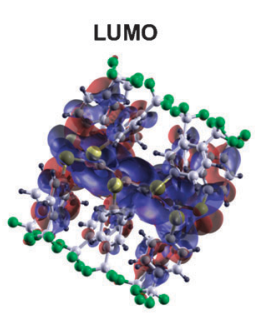

(b)

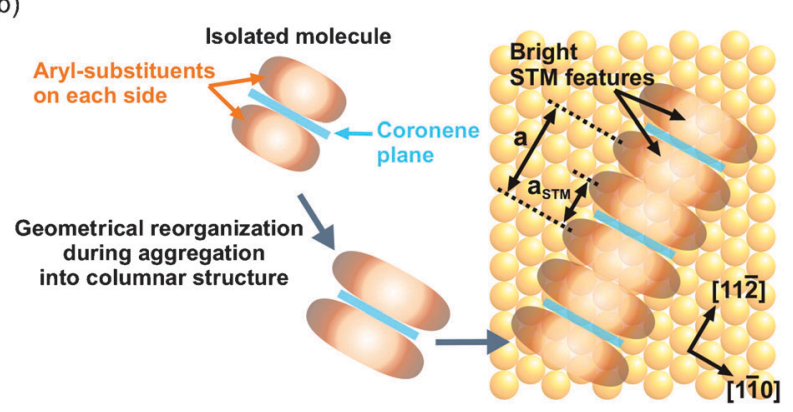

Fig. 6 Schematic drawing showing the supposed arrangement of DPTC and DFPTC molecules within the columnar structure on $\mathrm{Au}(111)$. (a) The molecules have adopted a conformation with an alternating pattern of the substituents above and below the coronene core, i.e. a conformation close to up-down or $2 u p-2 d o w n$, and lie on the surface in an edge-on configuration (coronene core perpendicular to the surface plane). (b) The columns grow along the [112]] direction and the intracolumnar separation between neighbouring molecules amounts to $a=2 \sqrt{ } 3 a_{\mathrm{Au}(111)}$. Due to protruding aryl groups on each side of the coronene core, bright features are observed in the STM images at almost equidistant separations of $a / 2 \approx a_{\mathrm{STM}}$. 
respectively. The greater intercolumnar distance of DFPTC is due to the additional space requirement of the $\mathrm{CF}_{3}$ groups. To allow a stacking with an intermolecular separation $a=$ $2 \sqrt{ } 3 a_{\mathrm{Au}(111)}$, the aryl groups of adjacent molecules in the columns have to interdigitate to a certain extent. To bring about the required space for this interdigitation, the arylthiosubstituents have to move away from the coronene core, i.e. the $\mathrm{C}-\mathrm{S}-\mathrm{C}$ bond angle increases. This widening and the associated increased diameter of the stacked molecules compared to the isolated ones is certainly larger in the case of DFPTC. Furthermore, the interdigitation of the aryl groups is expected to be accompanied by the formation of $\mathrm{C}-\mathrm{F} \cdots \pi$ interactions, which are typical intermolecular interactions between $\mathrm{CF}_{3}$ groups and trifluoromethyl substituted aromatic rings. ${ }^{23}$ Such additional attractive forces in the case of DFPTC might also lead to the enhanced tendency to form columnar stacks when compared to DPTC.

The kind of stacking within the columnar DATC arrangement is quite different from that of planar PAHs ${ }^{24}$ since the polyaromatic cores of the molecules cannot be stacked as densely as in dimers of such PAHs due to the steric hindrance of the arylthio groups. However, a comparatively strong interaction between the molecules in the columnar structure can be expected as a consequence of the strong interdigitation of aryl groups of adjacent molecules.

\subsection{Spectroscopic characteristics and electronic function}

Depending on the structural arrangement of the DATC molecules, we experimentally observed different electron transport characteristics. STS spectra for three different cases of DFPTC molecules are shown in Fig. 7. When the STM-tip is positioned above DFPTC molecules scattered around within the disordered regions, the normalized differential conductance (ndc) shows pronounced peaks in the positive and negative voltage region. Depending on the topographic appearance of the molecule, the spectra show pronounced differences. Fig. 7(a) shows the spectrum of molecules with a spherical shape and apparent diameters of about $1.5 \mathrm{~nm}$. The peaks in the ndc at positive and negative voltages are assigned to tunneling through the HOMO and LUMO states, respectively. The peak positions are varying only slightly for different molecules, giving a measured HOMO-LUMO transport gap of $\Delta E_{\mathrm{STS}} \approx 2.4 \mathrm{eV}$. On the other hand, molecules with a larger lateral size $(\sim 2 \mathrm{~nm})$ and a three-fold symmetry are observed in the STM images (Fig. 7(b)). Three bright intramolecular features with a centre to centre separation of $\sim 1.1 \mathrm{~nm}$ can be seen. The measured energetic gap in this case is considerably reduced. Here, the STS data yield a value of $\Delta E_{\mathrm{STS}} \approx 1.6 \mathrm{eV}$.

The HOMO-LUMO gap measured by STS can be written as $\Delta E_{\mathrm{STS}}=(\mathrm{IP}-\mathrm{EA}) /(1-\eta)$, where IP and EA are the ionization potential and electron affinity of the adsorbed molecules. ${ }^{25}$ Here, $\eta$ corresponds to the fraction of the total bias voltage $V$ which drops between the molecule and the substrate, ${ }^{26}$ i.e. a very small value of $\eta$ corresponds to a molecular state in close vicinity to the substrate. In addition, we assume that the molecules are only bound to the substrate by weak van der Waals interactions, as suggested by the diffusibility of DFPTC on the Au(111) surface at RT. Then, by applying the abovementioned relation, the
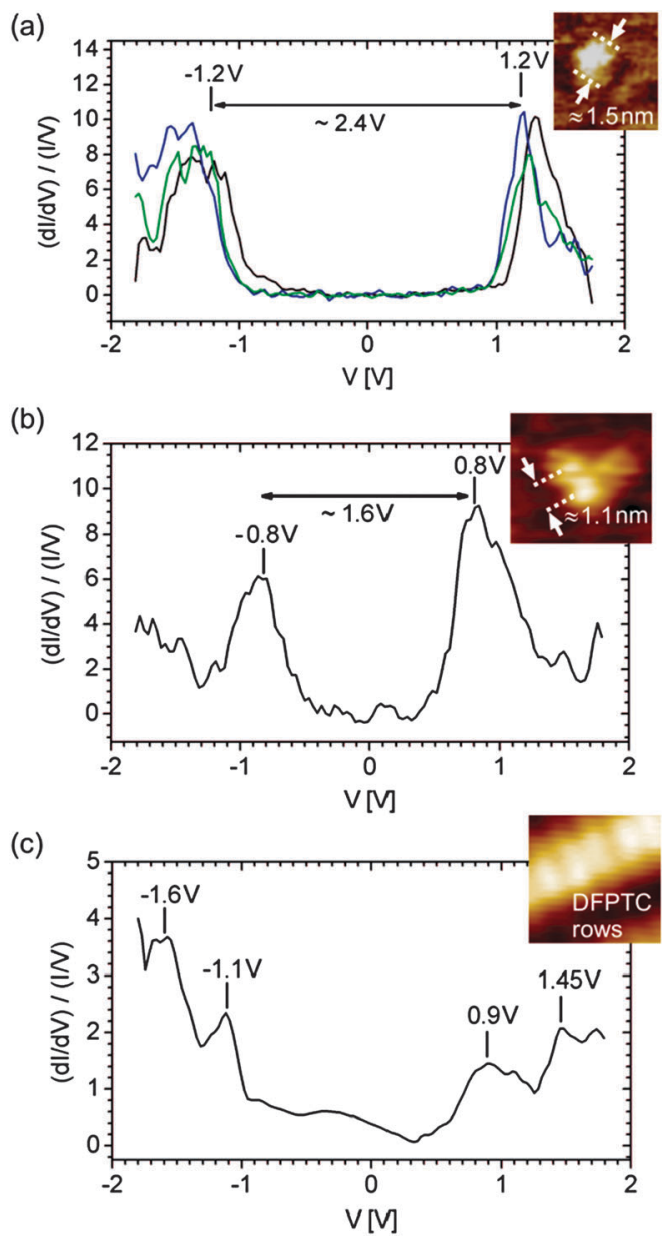

Fig. 7 STS data of DFPTC on $\mathrm{Au}(111)$. The topographic appearance of the molecules or molecular structure, on which the spectra were recorded, is depicted for each characteristic by a corresponding STM image. All shown negative differential conductance (ndc) versus voltage curves were recorded with spectroscopy set point values of $V_{\mathrm{s}}=1.3 \mathrm{~V}, I_{\mathrm{t}}=0.1 \mathrm{nA}$. (a) Single DFPTC molecules with spherical shape and an apparent diameter of $\sim 1.5 \mathrm{~nm}$. (b) Single DFPTC molecules with a threefold symmetry. (c) STS characteristics measured on top of the columnar DFPTC structure.

experimentally obtained energy gaps can be compared to results from our ab initio calculations of the electronic structure of single molecules in the gas phase. From the ground state total energies of the anion $\left(E_{\mathrm{tot}}(N+1)\right)$, neutral $\left(E_{\mathrm{tot}}(N)\right)$ and cation $\left(E_{\mathrm{tot}}(N-1)\right)$ species, we deduced the corresponding ionization potential (IP $\left.=E_{\mathrm{tot}}(N-1)-E_{\mathrm{tot}}(N)\right)$ and electron affinity $\left(\mathrm{EA}=E_{\mathrm{tot}}(N)-E_{\mathrm{tot}}(N+1)\right)$ of the different conformers, from which the fundamental energy $\operatorname{gap}^{27} \Delta E_{\text {theo }}=$ IP - EA is obtained. The calculated data and comparisons to experimental values are summarized in Table 2.

Several important statements should be noted: (i) the topographic appearance of a fraction of observed single DFPTC molecules (Fig. 7(a)) within the disordered layer fits best to the DFT geometry of the up-down conformer, (ii) the corresponding energy gap measured by STS, $\Delta E_{\mathrm{STS}}=(\mathrm{IP}-\mathrm{EA}) /$ $(1-\eta) \approx 2.4 \mathrm{eV}$, agrees very well with the theoretical results of the up-down conformer, considering the possible occurrence of a small voltage drop at the molecule-substrate interface, 
Table 2 Fundamental energy gaps in $\mathrm{eV}$ of the different DATC conformers

\begin{tabular}{lllll}
\hline & Up-down & 2up-2down & $U p-u p$ & $\Delta E_{\mathrm{STS}} / \mathrm{eV}$ \\
\hline DFPTC & 2.20 & 1.31 & 1.86 & $2.4^{a} / 1.6^{b}$ \\
DPTC & 2.24 & 1.34 & 1.85 & 2.2 \\
DMPTC & 2.16 & 1.05 & 1.40 & $2.96^{c} / 2.75^{d} / 2.20^{e}$
\end{tabular}

${ }^{a}$ Spherically shaped DFPTCs with a size comparable to the $u p$-down conformer. ${ }^{b}$ DFPTC with threefold symmetry and a size comparable to the 2 up-2down conformer. ${ }^{c} V_{\mathrm{s}}=1.3 \mathrm{~V}, I_{\mathrm{t}}=0.65 \mathrm{nA}(\eta=0.26)$. ${ }^{d} V_{\mathrm{s}}=1.3 \mathrm{~V}, I_{\mathrm{t}}=0.08 \mathrm{nA}(\eta=0.20) .{ }^{e}$ Extracted for $\eta=0$, i.e. for a very large tip-sample distance.

i.e. a non-zero value of $\eta$. (iii) DFPTC molecules with a threefold symmetric appearance (Fig. 7(b)) can be assigned to the 2up-2down conformer since the experimentally measured intramolecular features fit almost perfectly to the three pairs of protruding aryl groups above the plane of the coronene core (top view in Fig. 5(b)), and (iv) the theoretical energetic gap of the 2up-2down conformer agrees also reasonably well to the measured characteristics (for comparison see Table 1). Transitions between these stable conformations and intermediate conformations are also probable.

In turn, tunnelling spectra on top of DFPTCs within the columnar structure (Fig. 7(c)) exhibit distinctly different features when compared to the characteristics of single nonaggregated molecules, both the spherically shaped and the threefold symmetric ones. Here, a set of stabilized and destabilized HOMO/LUMO levels are observed. The change of the spectroscopic characteristics may have different origins. First of all, the stacking into the columnar structure is expected to go along with geometrical and therewith electronic reorganizations of the molecules. Furthermore, the columns grow commensurable to the $\mathrm{Au}(111)$ surface. This alignment to the substrate can be accompanied by molecule-substrate interactions and a mixing of electronic states located at the molecule and the metallic surface can be assumed on a limited scale. ${ }^{28,29}$ However, a decisive factor for the modification of the spectroscopic characteristics is probably the existence of electronic interactions between adjacent molecules of the columns. This is suggested by the fact that electrons are delocalized throughout the whole molecular system in the isolated DFPTC and also DPTC molecules. ${ }^{7,8}$ We have proven the delocalization of the frontier molecular orbitals by ab initio calculations. To exemplify, the HOMO of the up-down DFPTC conformer is shown in the middle panel of Fig. 6(a). Intermolecular $\pi-\pi$-interactions are suggested by the close proximity of the molecules in the columnar structure and the associated interdigitation of the peripheral aryl groups. Such a $\pi-\pi$-stacking motif is quite different from that of planar PAH systems having an intermolecular electronic overlap due to the close proximity of the polyaromatic cores. ${ }^{30-32}$ Since charges can be delocalized over the whole aromatic system of the DFPTC molecule, the overlap between the peripheral groups within the DFPTC columns should enable charge delocalization and thus lead to the build up of one-dimensional charge transport channels.

An opposed behaviour is observed in the case of the DMPTC molecules adsorbed face-on on $\mathrm{Au}(111) .^{32}$ In particular, a pronounced decoupling of the three-dimensional aromatic system from the environment, i.e. the substrate and surrounding molecules, is recognized and leads to another interesting electron transport characteristic. As shown in the STS spectrum in Fig. 8(a), the measured transport gap $\Delta E_{\mathrm{STS}} \approx$ $2.75 \mathrm{eV}$ is considerably larger when compared to non-aggregated DFPTCs or DPTCs. ${ }^{21}$ Furthermore, a significant $^{2}$ dependence of the energetic gap on the tip-sample distance is observed experimentally (Table 2). By applying the relation $\Delta E_{\mathrm{STS}}=(\mathrm{IP}-\mathrm{EA}) /(1-\eta)$, we deduce a value of $\Delta E_{\mathrm{STS}} \approx$ $2.2 \mathrm{eV}$ for the limit of $\eta=0$, i.e. for a very large tip-sample distance. The comparison to the theoretical fundamental gaps shows that this result fits nicely to the value of the most stable DMPTC up-down conformer (Tables 1 and 2).

Additionally, the STS spectra (Fig. 8(a)) exhibit a multiple peak structure. Subsequent to the first peaks at positive (peak labelled $N)$ and negative $(N+1)$ voltages, respectively, additional peaks are observed (denoted as $N-1$ for $V<0$ and $N+2, N+3$ for $V>0$ ). All energy differences (between the peaks $N+1 \rightarrow N+2, N+2 \rightarrow N+3$, and $N \rightarrow N-1$ ) are identical for a fixed tip-sample separation. Every pronounced STS peak can be ascribed to the opening of a new tunnelling channel when an additional charge state passes into the transport window. Therefore, the impact of the methoxyfunctionalization is twofold. Firstly, these groups seem to anticipate a stacking of the molecules and, secondly, they point towards the surface and therewith act as distance defining (insulating) spacers giving rise to a tunneling barrier between the substrate and the three-dimensional aromatic/delocalized electronic system of the molecules (Fig. 8(b)). A double-barrier tunnel junction is formed in this way and leads to the occurrence of single electron tunnelling effects in STS measurements. ${ }^{22}$

(a)

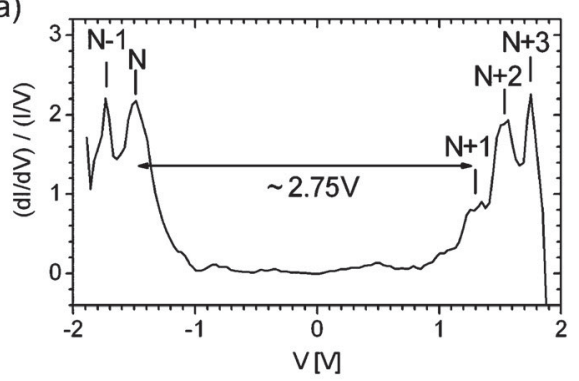

(b)

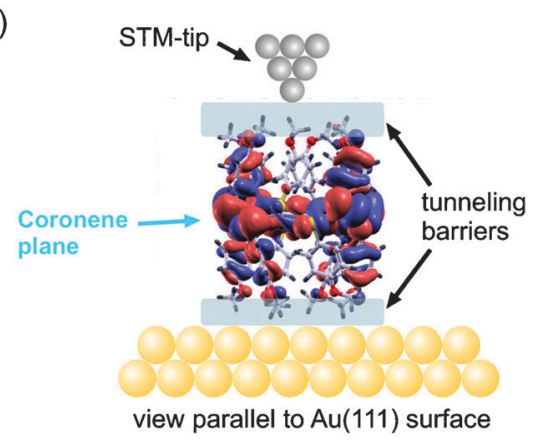

Fig. 8 (a) In the case of DMPTC adsorbed on Au(111) in a face-on configuration, the STS data show peaks related to subsequent charge states of the molecule. (b) Sketch showing that the methoxy groups pointing towards the substrate surface act as spacers, giving rise to an additional tunnelling barrier between the substrate and the threedimensional aromatic system of the DMPTC molecule. 


\section{Conclusions}

Per-substitution of a coronene core with phenylthio-ligands results in a three-dimensional aromatic system delocalized over the whole molecule verified by ab initio calculations. By using systematically varied arylthio-moieties with different substituents in the para-position, the self-assembly behaviour was directed into different regimes. Thus the tendency to form columnar structures of molecules in an edge-on configuration was strongly enhanced when using electron-withdrawing $\mathrm{CF}_{3}$ substituents at the peripheral arylthio moieties (DFPTC molecule). Accordingly, electron-donating $\mathrm{OCH}_{3}$ groups of the DMPTC molecule anticipated a stacking. Here, the molecules are adsorbed on the surface with a face-on orientation. This behaviour is comparable to the behaviour of PAHs despite the novel packing motif compared to the $\pi$ - $\pi$-stacking of planar PAH systems.

Furthermore, it was possible to identify different stable conformations of the adsorbed non-aggregated molecules by comparing STM/STS data with DFT results of the molecular geometry and electronic structure. It was demonstrated that the different adsorption geometries go along with a change in the electron transport characteristics. Moreover, DFPTCs assembled into a columnar structure showed distinct changes in the electronic structure. Along with the theoretically verified delocalization of the frontier molecular orbitals over the whole molecules, this modification of the spectroscopic characteristics points to the existence of electronic interactions between adjacent molecules in the columns.

The face-on adsorption of DMPTC together with not fully delocalized molecular orbitals, on the other hand, is accompanied by an electronic decoupling of the aromatic system from the substrate. In turn, this goes along with the creation of a double-barrier tunnel junction configuration and the occurrence of single electron transport features visible in the tunnelling spectroscopy. In conclusion, the investigated DATC system can be tailored in such a way that quite different advantageous electronic functionalities are achieved.

\section{Acknowledgements}

M.G. acknowledges the French National Center for Scientific Research (CNRS), Aix-Marseille II University and the University of Nice-Sophia Antipolis. The computations were performed under the auspices of the VSR at the computer JUROPA and the Gauss Centre for Supercomputing at the high-performance computer JUGENE operated by the Jülich Supercomputer Centre at the Forschungszentrum Jülich.

\section{References}

1 G. Maruccio, R. Cingolani and R. Rinaldi, J. Mater. Chem., 2004, 14, 542 .

2 N. J. Tao, Nat. Nanotechnol., 2006, 1, 173.

3 Y. Q. Xue and M. A. Ratner, Int. J. Quantum Chem., 2005, 102, 911

4 J. L. Bredas, J. P. Calbert, D. A. da Silva and J. Cornil, Proc. Natl. Acad. Sci. U. S. A., 2002, 99, 5804.

5 E. W. Meijer and A. P. H. J. Schenning, Nature, 2002, 419, 353.

6 M. Gingras, J. M. Raimundo and Y. M. Chabre, Angew. Chem., Int. Ed., 2006, 45, 1686.

7 J. H. R. Tucker, M. Gingras, H. Brand and J. M. Lehn, J. Chem. Soc., Perkin Trans. 2, 1997, 1303.

8 M. Gingras, A. Pinchart, C. Dallaire, T. Mallah and E. Levillain, Chem.-Eur. J., 2004, 10, 2895.

9 T. Braid, J. H. Gall, D. D. MacNicol, P. R. Mallinson and C. R. Michie, J. Chem. Soc., Chem. Commun., 1988, 1471.

10 B. Lüssem, S. Karthäuser, H. Haselier and R. Waser, Appl. Surf. Sci., 2005, 249, 197.

11 G. Kresse and J. Hafner, Phys. Rev. B: Condens. Matter, 1993, 47, 558.

12 G. Kresse and J. Furthmüller, Phys. Rev. B: Condens. Matter, 1996, 54, 11169

13 P. E. Blöchl, Phys. Rev. B: Condens. Matter, 1994, 50, 17953.

14 G. Kresse and D. Joubert, Phys. Rev. B: Condens. Matter, 1999, 59, 1758 .

15 J. P. Perdew, K. Burke and M. Ernzerhof, Phys. Rev. Lett., 1996, 77, 3865.

16 S. Grimme, J. Comput. Chem., 2006, 27, 1787.

17 N. Atodiresei, V. Caciuc, P. Lazic and S. Blügel, Phys. Rev. Lett., 2009, 102, 136809.

18 S. Sergeyev, W. Pisula and Y. H. Geerts, Chem. Soc. Rev., 2007, 36, 1902.

19 E. J. Foster, R. B. Jones, C. Lavigueur and V. E. Williams, J. Am. Chem. Soc., 2006, 128, 8569.

20 F. Cozzi, M. Cinquini, R. Annuziata and J. S. Siegel, J. Am. Chem. Soc., 1993, 115, 5330.

21 P. Kowalzik, S. Rathgeber, S. Karthäuser, R. Waser, N. Schnaebele, J.-M. Raimundo and M. Gingras, New J. Chem., 2012, DOI: $10.1039 / \mathrm{clnj} 20472 \mathrm{f}$.

22 P. Kowalzik, N. Atodiresei, M. Gingras, V. Caciuc, S. Blügel, R. Waser and S. Karthäuser, J. Phys. Chem. C, 2011, 115, 9204.

23 F. Emmerling, I. Orgzall, B. Dietzel, B. W. Schulz, G. Reck and B. Schulz, J. Mol. Struct., 2007, 832, 124.

24 I. D. Mackie and G. A. DiLabio, J. Phys. Chem. A, 2008, 112, 10968.

25 Y. Selzer and D. L. Allara, Annu. Rev. Phys. Chem., 2006, 57, 593.

26 S. Hong, R. Reifenberger, W. Tian, S. Datta, J. Henderson and C. P. Kubiak, Superlattices Microstruct., 2000, 28, 289.

27 S. Kummel and L. Kronik, Rev. Mod. Phys., 2008, 80, 3.

28 R. Temirov, S. Soubatch, A. Luican and F. S. Tautz, Nature, 2006, 444, 350 .

29 N. Gonzalez-Lakunza, I. Fernandez-Torrente, K. J. Franke, N. Lorente, A. Arnau and J. I. Pascual, Phys. Rev. Lett., 2008, 100, 156805 .

30 D. Adam, P. Schumacher, J. Simmerer, L. Haussling, K. Siemensmeyer, K. H. Etzbach, H. Ringsdorf and D. Haarer, Nature, 1994, 371, 141.

31 X. Feng, V. Marcon, W. Pisula, M. R. Hansen, J. Kirkpatrick, F. Grozema, D. Andrienko, K. Kremer and K. Müllen, Nat. Mater., 2009, 8, 421.

32 D. Käfer, A. Bashir, X. Dou, G. Witte, K. Müllen and C. Wöll, Adv. Mater., 2009, 21, 1 